notes de lecture

\title{
Les écoles et leur réputation
}

Hugues Draelants \& Xavier Dumay (Dir.), Louvain-la-Neuve, De Boeck, 2016

Xavier Pons

\section{OpenEdition \\ Journals}

Édition électronique

URL : https://journals.openedition.org/ries/5775

DOI : $10.4000 /$ ries. 5775

ISSN : 2261-4265

Éditeur

France Education international

Édition imprimée

Date de publication : 1 avril 2017

Pagination : $32-33$

ISBN : 978-2-85420-614-2

ISSN : $1254-4590$

\section{Référence électronique}

Xavier Pons, «Les écoles et leur réputation », Revue internationale d'éducation de Sèvres [En ligne], 74 | avril 2017, mis en ligne le 01 avril 2017, consulté le 11 mars 2022. URL : http:// journals.openedition.org/ries/5775; DOI : https://doi.org/10.4000/ries.5775

Ce document a été généré automatiquement le 11 mars 2022.

(c) Tous droits réservés 


\title{
Les écoles et leur réputation
}

\author{
Hugues Draelants \& Xavier Dumay (Dir.), Louvain-la-Neuve, De Boeck, \\ 2016
}

\section{Xavier Pons}

1 Collectif, comparatif et plus empirique, cet ouvrage s'inscrit dans le prolongement de celui sur l'«identité des établissements scolaires" publié cinq plus tôt par Hugues Draelants et Xavier Dumay aux Presses universitaires de France. D'ailleurs, il s'agit moins ici de travailler la notion de "réputation " en tant que telle, que de continuer à réfléchir à la construction de ces identités d'établissement et à leurs rôles et fonctions sur les marchés scolaires. Les auteurs entendent ainsi nuancer « l'idée selon laquelle la construction des identités [d'établissement] se réduirait à un travail de façade. La «marketisation» des établissements est une réalité; cependant le souci de la réputation n'entraîne pas nécessairement un fort découplage entre l'image projetée et le fonctionnement interne des établissements"(p.9). Il s'agit donc de mieux comprendre le processus de fabrication de ces identités et leur effet sur l'environnement des établissements.

2 La première partie de l'ouvrage propose ainsi huit études de cas qui illustrent divers aspects de ce processus de fabrication. Ces aspects sont plus ou moins convergents avec la revue de littérature présentée dans l'ouvrage de 2011. Cette dernière insiste sur le rôle moteur joué par les directions désireuses de mieux contrôler l'image de leur établissement. L'identité de ce dernier est alors essentiellement conçue comme le résultat d'une stratégie de maîtrise des «impressions organisationnelles " qui s'en dégagent, donc selon une approche plutôt instrumentaliste. Dans cette première partie, au contraire, les études de cas partagent une approche plus constructiviste et relationnelle de l'identité, selon laquelle l'identité d'un établissement se construit selon une triple relation : à lui-même (son histoire, ses membres etc.), à son environnement et aux valeurs et aux normes du champ institutionnel dans lequel il est encastré.

3 À partir d'entretiens et de divers documents internes à un établissement belge francophone étudié dans la durée (1977-2008), A.-F. Taymans et X. Dumay analysent dans quelle mesure les directions successives marquent de leur « empreinte » l'identité 
de cet établissement et concluent à une co-production de cette identité par les chefs d'établissement et d'autres acteurs. B. Darchy-Koechlin et H. Draelants étudient le travail de perpétuation de la mémoire et du prestige effectué par certaines grandes écoles françaises dans un contexte d'internationalisation de l'enseignement supérieur. Dans un troisième chapitre, H. Draelants et X. Dumay comparent deux établissements belges contrastés en termes de réputation et de résultats: ils montrent que la réputation de ces établissements est tenace malgré le travail effectué dans un établissement difficile pour obtenir de meilleurs résultats ou la crise que peut rencontrer un établissement prestigieux. V. Dupriez et R. Wattiez, pour leur part, analysent la concurrence que se livrent des établissements belges d'un même bassin de recrutement. Ils mettent en évidence que cet espace local est structuré en différentes « niches éducatives » et que la concurrence a lieu essentiellement au sein d'une même niche.

Les deux chapitres suivants se concentrent sur l'impact des transformations des modes de régulation externes sur l'identité des établissements. S. Bar, X. Dumay et H. Draelants reviennent sur les effets des classements d'établissements anglais sur les relations que ceux-ci entretiennent avec les communautés locales. Ils montrent que ces classements ont une influence importante sur la manière dont les établissements se définissent, malgré les efforts déployés par les directions pour limiter la prise en compte de ces classements par ailleurs volatiles. C. Lejeune et A. Vas aboutissent à une conclusion similaire concernant les effets des procédures d'accréditation des écoles de commerce françaises. À partir de l'étude de trois écoles n'ayant pas obtenu du premier coup l'accréditation EQUIS ${ }^{1}$, ils mettent en évidence que cette procédure déclenche un changement identitaire radical de ces écoles souvent sous-estimé par les écoles impliquées. Les deux chapitres qui suivent proposent un raisonnement inverse en montrant le rôle de certains établissements dynamiques dans le renouvellement des identités d'établissement en présence en Communauté française de Belgique : E. Jadot, C. Theys et M. Verhoeven illustrent ce processus à partir de l'exemple des «écoles citoyennes » et J. Bachiri, G. Ramis et X. Dumay à partir du cas du réseau d'écoles islamiques.

5 La deuxième partie de l'ouvrage envisage deux effets principaux de ces identités d'établissement sur leur environnement. D'une part, ces identités fonctionnent comme des "systèmes de simplification", qui permettent aux établissements de trier les demandes qui leur sont adressées par les parents ou l'institution, de les hiérarchiser et de les ajuster à leur mode de fonctionnement privilégié. C'est ce qu'illustrent A. Carrasco, C. Flores et G. Guttiérez dans leur analyse des pratiques sélectives des écoles primaires prestigieuses au Chili ou S. Barbana quand il montre que les «bonnes écoles » belges peuvent plus facilement résister à la pression introduite par des épreuves d'évaluation certificative externe.

6 D'autre part, ces identités fonctionnent comme des «dispositifs de jugement et d'appariement » sur les marchés scolaires. J. Deceuninck et H. Draelants montrent, par exemple, que la réforme des inscriptions à l'entrée de l'enseignement secondaire en Belgique francophone, qui introduit une procédure plus bureaucratique que le fonctionnement précédent, a pour effet de standardiser l'appariement et, paradoxalement, de donner une place déterminante aux réputations des établissements. A van Zanten et A. Ollivier, enfin, mettent en évidence que les journées portes ouvertes et autres initiatives des établissements d'enseignement supérieur 
français envers leur public potentiel ont pour fonction de construire un rapport d'équivalence et de connivence entre les établissements et leurs visiteurs, qui influe sur les procédures de choix de ces établissements par les étudiants et leurs familles. S'opère alors un " appariement statutaire ", sur la base de ces identités.

7 Comme en témoigne cette longue énumération, cet ouvrage est particulièrement riche et a le grand mérite, par un cadrage conceptuel clair, de s'inscrire de manière cumulative dans plusieurs traditions de questionnement sur l'évolution des modes de régulation des systèmes scolaires.

\section{NOTES}

1. . European Quality Improvement System.

\section{AUTEUR}

\section{XAVIER PONS}

Xavier Pons est maître de conférences à l'Université Paris-Est Créteil (UPEC), chercheur au Laboratoire interdisciplinaire d'études du politique - Institut Hannah Arendt (EA 7373) et chercheur associé à l'Observatoire sociologique du changement (OSC-Sciences Po). Membre de plusieurs projets de recherche comparatifs depuis 2006, ses travaux de sociologie de l'action publique et des politiques éducatives portent principalement sur les transformations des modes de gouvernance des systèmes éducatifs, les réformes des administrations scolaires, les transformations des États éducateurs en Europe et les modes de structuration du débat public en éducation. Lauréat 2011 du Prix Jean-Claude Eicher pour le développement de la recherche en éducation, il est l'auteur de plusieurs articles et ouvrages sur ces thèmes. Courriel : ponsx@wanadoo.fr 